\title{
Interplay of mesoscopic and Kondo effects for transmission amplitude of few-level quantum dots
}

\author{
T. Hecht, ${ }^{1}$ A. Weichselbaum, ${ }^{1}$ Y. Oreg, ${ }^{2}$ and J. von Delft ${ }^{1}$ \\ ${ }^{1}$ Physics Department, Arnold Sommerfeld Center for Theoretical Physics and Center for NanoScience, \\ Ludwig-Maximilians-Universität München, Germany \\ ${ }^{2}$ Department of Condensed Matter Physics, The Weizmann Institute of Science, Rehovot 76100, Israel
}

(Dated: October 25, 2018)

\begin{abstract}
The magnitude and phase of the transmission amplitude of a multi-level quantum dot is calculated for the mesoscopic regime of level spacing large compared to level width. The interplay between Kondo correlations and the influence by neighboring levels is discussed. As in the single-level case, the Kondo plateaus of magnitude and phase disappear with increasing temperature. At certain gate voltages, "stationary" points are found at which the transmission phase is independent of temperature. Depending on the mesoscopic parameters of the adjacent levels (like relative sign and magnitude of tunneling matrix elements), the stationary points are shifted to or repelled by the neighboring level.
\end{abstract}

PACS numbers: 73.23.Hk, 73.23.-b, 73.63.Kv, 73.40.Gk

\section{INTRODUCTION}

In a remarkable series of experiments, $,+2,3,4,5,6$ the Heiblum group has analyzed the complex transmission amplitude, $t_{d}=\left|t_{d}\right| e^{i \alpha}$, of a quantum dot embedded in an Aharonov-Bohm ring. In particular, by analyzing the Aharonov-Bohm oscillations of the conductance of such a ring, the dependence of both the magnitude and phase of the transmission amplitude, $\left|t_{d}\right|$ and $\alpha$, were measured as a function of various parameters such as gate voltage $V_{g}$ applied to the dot, temperature $T$, mean coupling strength to the leads $\Gamma$, etc.

The first two experiments in this series, 1,2 dealt with large dots containing many $(>100)$ electrons. The experiment by Yacoby et al $\stackrel{1}{n}$ showed that coherent transport through a quantum dot is possible despite the presence of strong interactions. The next experiments by Schuster et $a l^{2} \stackrel{2}{*}$ generated tremendous interest because the behavior of the transmission phase showed a surprisingly "universal" behavior as function of gate voltage: the phase experienced a series of sudden jumps by $-\pi$ (phase lapses) between each pair of Coulomb blockade peaks in the conductance through the dot. This contradicted a naive expectation that the behavior of the transmission phase should depend on microscopic details of the dot, such as the signs of the matrix elements coupling a given level to the left or right lead.

Subsequent experiments by Ji et al. $\stackrel{3,4}{=}$ performed on smaller dots containing tens of electrons, analyzed how the occurrence of the Kondo effect influences the transmission amplitude, and in particular its phase. For transmission at zero temperature through a single level, the Kondo effect causes the magnitude of the transmission amplitude to exhibit (as function of gate voltage) a plateau at the unitary limit $\left(\left|t_{d}\right|=1\right)$. For this regime it had been predicted by Gerland et al $\underline{7}$ that the phase should show a plateau at $\alpha=\pi / 2$, a result very different from the universal behavior mentioned above. While the experiments of Ji et al. did yield deviations from the universal phase behavior, they did not verify the prediction of a $\pi / 2$ Kondo plateau in the phase. With hindsight, the reason probably was that the experiments did not realize the conditions assumed in the calculations of Gerland et al.,$\frac{7}{,}$ namely transport through only a single level.

Truly "mesoscopic" behavior for the phase was observed only rather recently by Avinun-Kalish et al.,$\underline{\underline{\underline{5}}}$ in even smaller dots containing only a small $(<10)$ number of electrons. For these, the mean level spacing $\delta$ was significantly larger than the average level width $\Gamma$, so that for any given gate voltage, transport through the dot is typically governed by the properties of only a single level, namely that closest to the Fermi energies of the leads. When the number of electrons was increased beyond about 14, universal behavior for the phase was recovered. Consequently, it was proposed ${ }^{5,8,9,10,11,12,13}$ that the universal behavior occurs whenever a quantum dot is large enough for that the ratio $\delta / \Gamma$ is sufficiently small $(\simeq 1)$ that for any given gate voltage, typically more than one level contributes to transport.

The latest paper in this series, by Zaffalon et al.,$\frac{6}{s}$ studied the transmission phase through a quantum dot in the "deep mesoscopic" regime $\delta / \Gamma \gg 1$, containing only one or two electrons. When this system was tuned into the Kondo regime, the transmission phase indeed did show the $\pi / 2$ Kondo plateau predicted by Gerland et al $\underline{\underline{7}}$.

The experiments of Avinun-Kalish et al. $\frac{5}{,}$ which observed mesoscopic effects for the transmission phase through a small number of levels, and those of Zaffalon et $a l ., \frac{6}{w}$ which found characteristic signatures of the Kondo effect in the transmission phase through a single level, raise the following question: what type of phase behavior can arise in the deep mesoscopic regime from the interplay of (i) random signs for tunneling amplitudes of neighboring levels and (ii) the Kondo effect for individual levels? In the present paper, we address this question by studying spin-degenerate models of dots with 2 or 3 levels in the deep mesoscopic regime of $\delta / \Gamma \gg 1$. This is the 
regime relevant for the experiments of Zaffalon et al $\underline{\underline{6}}$ (for those of Ji et al. 3.4 the ratio $\delta / \Gamma$ was presumably smaller than used here). Our goal is to provide a catalogue of the types of behavior that can occur in this regime, and to illustrate how the characteristic transmission amplitude (magnitude and phase) depends on temperate as well as on the strength of the coupling to the leads.

This paper is organized as follows. In Sec. II we introduce our many-level model for the quantum dot system. We discuss the relation between the AharonovBohm contribution to the linear conductance and the transmission amplitude through the quantum dot. The latter can be expressed in terms of the local Green's function of the dot. We briefly present the technique used to calculate the latter, the numerical renormalization group method. In Sec. III we present our numerical results of both the phase and the magnitude of the transmission amplitude through a two- and three-level model in the regime $\delta / \Gamma \gg 1$. We discuss the $T$ - and $\Gamma$-dependence of the transmission amplitude with focus on the influence on Kondo correlations. We study all relevant choices of the mesoscopic parameters given by the relative signs of the tunneling amplitudes of adjacent levels. The influence of neighboring levels is studied. It results not only in a phase lapse in Coulomb blockade valleys but also introduces a $V_{g}$-asymmetry in the finite temperature modulations of the Kondo plateaus. "Stationary" points of $T$ - and $\Gamma$-independence are discussed. In the Appendix, we give a derivation of a formula for the Aharonov-Bohm contribution to the linear conductance through a multiterminal interferometer with open geometry, as used in the Heiblum group. This formula has been used in several publications including some of the present authors, $,, 11,12$ but its derivation had not been published before.

\section{THE MODEL AND THE METHOD}

In the experiments, $, 2,3,4,5,6$ the temperature-dependent transmission amplitude through the quantum dot is extracted from the Aharonov-Bohm oscillations of the conductance in a multi-lead ring geometry. In the Appendix we show that this transmission amplitude can be expressed in terms of the equilibrium local Green's function of the dot tunnel-coupled only to two leads on its left and right side, without explicitly incorporating the other leads of the ring geometry in the calculation.

In this Section we introduce a "reduced model" describing the latter situation of a spinful multi-level quantum dot coupled to two reservoirs and present the transmission formula derived in the Appendix. Further, we comment on NRG, the method used to calculate the local Green's function.

\section{The model Hamiltonian}

The model Hamiltonian can be split into three parts,

$$
H=H_{\mathrm{d}}+H_{\mathrm{l}}+H_{\mathrm{t}},
$$

specifying the properties of the bare dot, the leads and the coupling between the two systems, respectively. For $N$ spinful levels coupled to a left (emitter) and right (collector) lead, these terms are given by

$$
\begin{aligned}
H_{\mathrm{d}} & =\sum_{j=1 . . N} \sum_{\sigma} \varepsilon_{d j} n_{d j \sigma}+\sum_{\{j \sigma\} \neq\left\{j^{\prime} \sigma^{\prime}\right\}} U n_{d j \sigma} n_{d j^{\prime} \sigma^{\prime}} \\
H_{\mathrm{l}} & =\sum_{\alpha=L, R} \sum_{k \sigma} \varepsilon_{k} c_{\alpha k \sigma}^{\dagger} c_{\alpha k \sigma} \\
H_{\mathrm{t}} & =\sum_{j} \sum_{\alpha=L, R} \sum_{k \sigma}\left(t_{\alpha}^{j} c_{\alpha k \sigma}^{\dagger} d_{j \sigma}+\text { H.c. }\right) .
\end{aligned}
$$

Dot creation operators for level $j$ and spin $\sigma=\{\uparrow, \downarrow\}$ are denoted by $d_{j \sigma}^{\dagger}$, with $n_{d j \sigma}=d_{j \sigma}^{\dagger} d_{j \sigma}$, where $j=$ $1 \cdots N$ labels the levels in order of increasing energy $\left(\varepsilon_{d j}<\varepsilon_{d j+1}\right)$. We use an inter- and intra-level independent Coulomb energy $U>0$. The leads are assumed to be identical and non-interacting with constant density of states $\rho=1 / 2 D$, where the half-bandwidth $D=1$ serves as energy unit. Electrons in lead $\alpha$ are created by $c_{\alpha k \sigma}^{\dagger}$. The local levels are tunnel-coupled to the leads, with real overlap matrix elements $t_{\alpha}^{j}$ that for simplicity we assume to be energy- and spin-independent. The resulting broadening of each level is given by $\Gamma_{j}=$ $\Gamma_{j L}+\Gamma_{j R}$, with $\Gamma_{j \alpha}=\pi \rho\left(t_{\alpha}^{j}\right)^{2}$. Notation: We define $s_{i}=\operatorname{sgn}\left(t_{L}^{i} t_{R}^{i} t_{L}^{i+1} t_{R}^{i+1}\right)= \pm$. For example, matrix elements of same sign result in $s_{i}=+$, whereas one different sign yields $s_{i}=-$. We further define $s \equiv\left\{s_{1} \cdots s_{N-1}\right\}$, and use $\gamma=\left\{\Gamma_{1 L}, \Gamma_{1 R}, \cdots, \Gamma_{N L}, \Gamma_{N R}\right\} / \Gamma$, with the mean level broadening $\Gamma=1 / N \sum_{j} \Gamma_{j}$. We assume constant level spacing $\delta=\varepsilon_{d i+1}-\varepsilon_{d i}$. The local levels can be shifted in energy by a plunger gate voltage $V_{g}$, with $\varepsilon_{d j}=j \delta-\left(V_{g}+V_{g 0}\right)$, where $V_{g 0}=\frac{N-1}{2} \delta+\frac{2 N-1}{2} U$. This convention ensures that in case of maximal symmetry $\left(t_{\alpha}^{j}=\right.$ const. for all $\left.j, \alpha\right)$, the system possesses particlehole symmetry at $V_{g}=0$.

\section{Transmission}

In the Appendix we generalize a result of Bruder, Fazio and Schoeller ${ }^{14}$ to show that the Aharonov-Bohm contribution to the linear conductance through the multiterminal interferometer with open geometry with a multilevel quantum dot embedded in one arm (see Fig. [ in the Appendix) can be expressed as

$$
G^{A B}(T)=\frac{e^{2}}{h}\left|T_{u}\right|\left|t_{d}(T)\right| \cos \left(2 \pi \Phi / \Phi_{0}+\phi_{0}+\alpha(T)\right) .
$$

Here $T_{u}=\left|T_{u}\right| e^{i \phi_{0}+i 2 \pi \Phi / \Phi_{0}}$ is the energy- and temperature-independent transmission amplitude 
through the upper reference arm including the AharonovBohm contribution $2 \pi \Phi / \Phi_{0}$ to the phase, where $\Phi$ is the magnetic flux enclosed by the interferometer arms and $\Phi_{0}=h / e$ is the flux quantum. The equilibrium Fermi function of the leads are denoted by $f_{0}$. The effective, temperature-dependent transmission amplitude $t_{d}(T)$ through the lower arm including the quantum dot is given by

$$
t_{d}(T)=\int d E\left(-\frac{\partial f_{0}(E, T)}{\partial E}\right) T_{d}(E, T) \equiv\left|t_{d}\right| e^{i \alpha},
$$

where

$$
T_{d}(E, T)=\sum_{j j^{\prime}} \sum_{\sigma \sigma^{\prime}} 2 \pi \rho t_{L}^{j} t_{R}^{j^{\prime}} \mathcal{G}_{j \sigma, j^{\prime} \sigma^{\prime}}^{R}(E, T) .
$$

Therefore, only local properties like the local retarded Green's function $\mathcal{G}_{j \sigma, j^{\prime} \sigma^{\prime}}^{R}$ and the Fermi function of the leads enter in the transmission amplitude through the quantum dot $t_{d}$ [Eq. (3)]. Thereby the local Green's function is evaluated for the model given in Eqs. (11) in equilibrium at temperature $T$.

In the zero temperature limit and in linear response, the dot produces purely elastic potential scattering between left and right leads, which can be fully characterized 15 by the eigenvalues $e^{i 2 \delta_{\nu}}(\nu=a, b)$ per spin of the $S$-matrix, and the transformation $\left(\begin{array}{cc}\cos \theta & \sin \theta \\ -\sin \theta & \cos \theta\end{array}\right)$, that maps the left-right basis of lead operators onto the $a-b$ eigenbasis of $S$. The transmission amplitude through the dot then reads

$$
t_{d}=-i S_{L R}=\sin (2 \theta) \sin \left(\delta_{a}-\delta_{b}\right) e^{i\left(\delta_{a}+\delta_{b}\right)},
$$

where in general $\theta$ and $\delta_{\nu}$ are all $V_{g}$-dependent. The phase $\delta_{\nu}$ is related by the Friedel sum rule $\frac{16}{6}$ to the charge (per spin) $n_{\nu}=\delta_{\nu} / \pi$ extracted by the dot from effective lead $\nu$. As $V_{g}$ is swept, the transmission amplitude goes through zero whenever $n_{a}=n_{b} \bmod 1$, and a phase lapse by $\pi$ occurs. Equation (5) is useful for the special case of "proportional couplings", $t_{L}^{j}= \pm \lambda t_{R}^{j}$ with $\lambda$ independent of $j$, in which the occupations $n_{a, b}$ take a simple form. Then the two effective leads $a$ and $b$ are the even and odd combinations of the left and right leads, respectively, with $\tan \theta=1 / \lambda$ independent of $V_{g}$. Then each level either couples to the even or the odd lead, and the occupations extracted from the leads are given by $n_{E, O}=\sum_{j \in E, O} n_{d j \sigma}$. Note that if all levels are coupled to the same effective lead (which is the case for $s=\{+\cdots+\}$ ), the other effective lead decouples, thereby reducing the computational complexity significantly.

\section{The method}

We calculate the local Green's function $\mathcal{G}^{R}$ needed for the transmission amplitude (Eqs. (3) and (41), respectively) using the numerical renormalization group $\operatorname{method}^{17}$ (NRG), a well-established method for the study of strongly correlated impurity systems. For a review, see Ref. 18. The key idea of NRG is the logarithmic discretization of the conduction band with a discretization parameter $\Lambda>1$. As a result, $H_{1}$ is represented as a semi-infinite chain, where only the first site couples to the local level. The hopping matrix elements along the chain fall off exponentially like $\Lambda^{-(n-1) / 2}$ with the site number $n$ (energy scale separation). The NRG Hamiltonian can be solved iteratively by successively adding sites and solving the enlarged system, thereby increasing the energy resolution with each added site by a factor of $\Lambda^{1 / 2}$. The corresponding increase in Hilbert space is dealt with by a truncation strategy that keeps only the lowest $N_{\text {keep }}$ states for the next iteration.

For the calculation of $\operatorname{Im} \mathcal{G}^{R}$ we use the full density matrix $\mathrm{NRG}^{19,20}$, based on the only recently developed concept of a complete basis set within NRG 21 . The real part of $\mathcal{G}^{R}$ is obtained by Kramers-Kronig transformation. Improvement of the results is obtained by the selfenergy representation, where the $U$-dependent part of the impurity self-energy $\Sigma(\omega)=U \frac{F^{R}(\omega)}{G^{R}(\omega)}$ is expressed by two correlation function ${ }^{22}$, which both are calculated with the full density matrix NRG.

\section{RESULTS}

In this Section we present our results for the phase and magnitude of the transmission amplitude $t_{d}$ through the quantum dot. The gate voltage $V_{g}$ is swept over a range sufficiently large that the full occupation spectrum of the quantum dot is covered ranging from 0 to $2 N$. The exact distribution of the couplings seems to play only minor role for the transmission amplitude. Therefore we choose left-right symmetric coupling in the cases where all $s_{j}=+$, reducing the computational effort significantly, since then the odd channel decouples.

In the regime of interest, the deep mesoscopic regime, the mean level spacing $\delta$ is much larger than the typical level widths $\Gamma_{j}, \delta / \Gamma \gg 1$. Therefore electrons enter the dot one by one when increasing the gate voltage. Transport thus occurs mainly through one level at a time; more precisely, it occurs through a linear combination of all levels, where in the mesoscopic regime the level closest to the Fermi energy dominates $\underline{\underline{11}}$

The Section is organized as follows: We first eludicate the basic properties of the transmission amplitude for the example of a two-level system. Varying temperature $T$ (at fixed coupling $\Gamma$ ), or average coupling $\Gamma$ (at fixed $T$ ), we study both possible choices $s=+$ and $s=-$, respectively. In order to analyze the interplay of $s=+$ and $s=-$, we then present data for a three-level system for all four possible combinations of $s_{1}, s_{2}$. Additionally, this has the advantage that for the middle level "boundary effects" (effecting the outermost levels) can be assumed to be eliminated, thus the behavior of the middle level can 


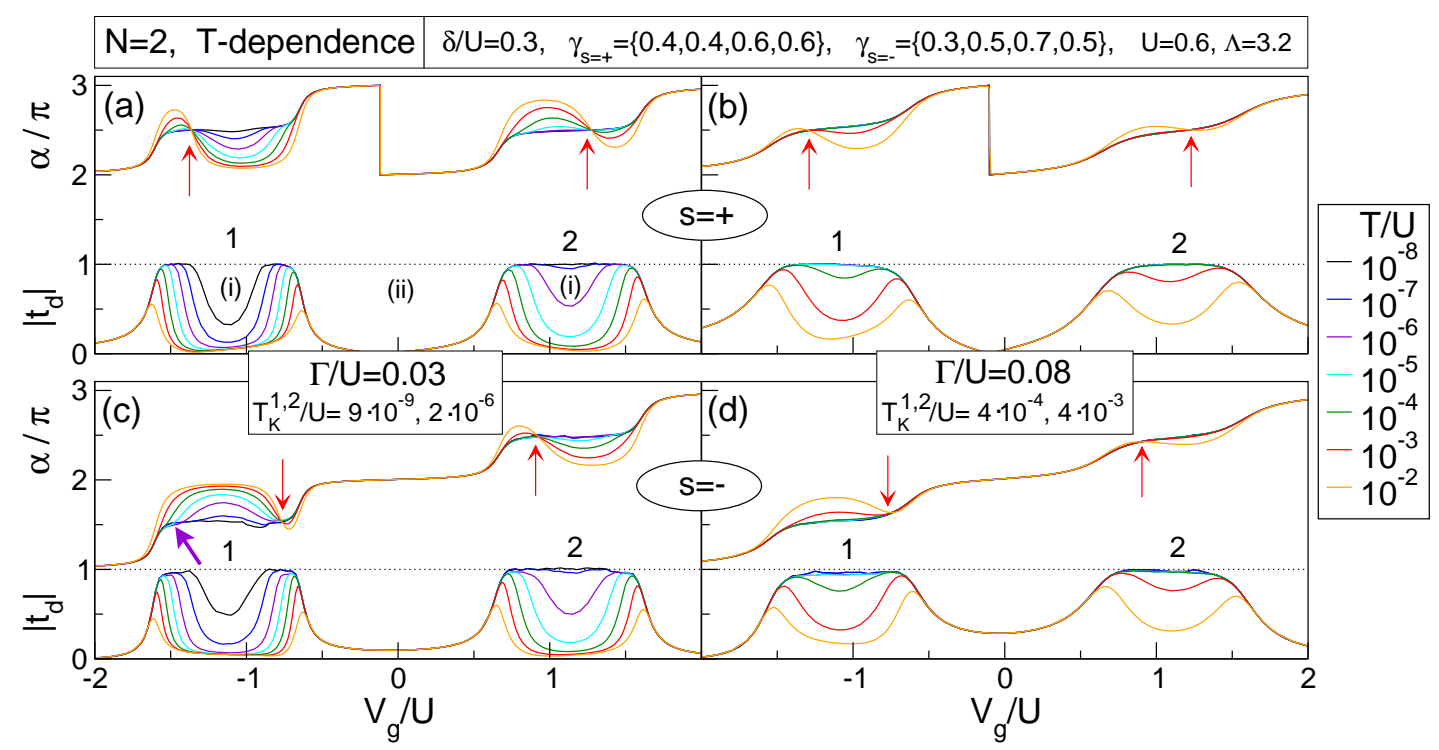

FIG. 1: Transmission $t_{d}=\left|t_{d}\right| e^{i \alpha}$ through a spinful two-level quantum dot for various temperatures and constant couplings. Regimes (i), (ii), indicated in panel (a) only, refer to Kondo valleys or Coulomb blockade valleys, respectively (see text). The levels involved are indicated by their level number 1,2. Level 2 is coupled more strongly to the leads than level 1 , resulting in different bare Kondo temperatures, e.g. $T_{K}^{j=1}>T_{K}^{j=2}$. We use $\Gamma / U=0.03(\mathrm{a}, \mathrm{c})$ and $\Gamma / U=0.08(\mathrm{~b}, \mathrm{~d})$, thus $T_{K}^{(a, c)}<T_{K}^{(b, d)}$. The minimum value of the $T_{K}^{j}$ (in the center of the Kondo valleys) are indicated in the legends. In accordance with Ref. 23 , we find shoulders in the phase (see e.g. the fat purple arrow and the purple curve $\left(T / U=10^{-6}\right)$ in $(\mathrm{c})$ for level 1$)$ and an enhanced sensitivity of the phase to Kondo correlations compared to the magnitude, see e.g. the green $\left(T / U=10^{-4}\right)$ curves in (d) for level 1 or the purple curve $\left(T / U=10^{-6}\right)$ for level 2 in (a). There, the typical $\frac{\pi}{2}$-Kondo plateau in the phase is present, whereas the Kondo plateau in amplitude is not fully developed yet. At certain points in gate voltage, say $V_{g}^{c_{j}}$ (as indicated by red arrows), we find stationary points where the curves for $\alpha$ for all temperatures intersect. The position of $V_{g}^{c_{j}}$ is shifted by the presence of a neighboring level, being repelled by or shifted towards the latter for $s=+$ or - , compare (a,c) or (b,d), respectively. Depending on the mesoscopic parameter $s= \pm$, the phase either exhibits a sharp drop of $\pi$, accompanied by a zero in the amplitude $\left|t_{d}\right|(s=+$, see $(\mathrm{a}, \mathrm{b}))$, or increases monotonically $(s=-$, see $(\mathrm{c}, \mathrm{d}))$ in the Coulomb blockade valleys.

be viewed as representative of a generic level in a multilevel quantum dot in the deep mesoscopic regime.

Unless otherwise noted, we use $U=0.6$. In order to cover all relevant energy scales with reasonable computational effort, we usually use $\Lambda=3.2$ for the two-level model and $\Lambda=3.5$ in case of three levels. We checked that already by keeping $\sim 1000$ states at each iteration, also for the two-channel calculations (that involve at least one $s_{i}=-$ ) the physical trends are captured qualitatively. Note that since the eigenvalues of the scattering matrix are given by $e^{i 2 \delta_{\nu}}$, the transmission phase $\alpha$ is defined modulo $\pi$. For clarity of the Figures, curves showing $\alpha$ are shifted by multiples of $\pi$ as convenient.

\section{A. Two-level model}

\section{Temperature dependence}

Figure 1 shows the transmission amplitude for both $s=+(\mathrm{a}, \mathrm{b})$ and $s=-(\mathrm{c}, \mathrm{d})$, for fixed dot parameters and various different temperatures. The mean level broadening is chosen to be $\Gamma / U=0.03$ in panels $(\mathrm{a}, \mathrm{c})$, and $\Gamma / U=0.08$ in panels $(\mathrm{b}, \mathrm{d})$. Therefore the $\left(V_{g^{-}}\right.$ dependent) bare Kondo temperatures

$$
T_{K}^{j}=\sqrt{\frac{\Gamma_{j} U}{2}} \exp \left[-\pi \frac{\varepsilon_{d j}}{2 U} \frac{\left(\varepsilon_{d j}+U\right)}{\Gamma_{j}}\right]
$$

vary in a lower-lying range of energies for panels $(a, c)$ than for panels $(b, d)$. In all panels the relative coupling of the first and the second levels are chosen to be $\gamma=\{0.8,1.2\}$. Therefore, the bare Kondo temperature for level 1 is lower than for level $2, T_{K}^{j=1}<T_{K}^{j=2}$, as indicated in the legends. The resulting difference in the temperature dependence can be nicely observed in the Figure. We first describe those general properties of the transmission amplitude that qualitatively agree with those that one would obtain for just a single level, then discuss the effect of the presence of a second level.

General properties: In the mesoscopic regime, where transport mainly occurs through one level at a time, two different regimes of transmission can be distinguished as $V_{g}$ is varied, as indicated in Fig. 1(a): (ii) In the regime between the Kondo valleys, to be called "Coulomb blockade valleys", the transmission amplitude is mainly determined by the mesoscopic parameter $s$, showing a phase lapse only in case $s=+$, similar for both spinful and 
spinless models 11,12 .

In the zero temperature limit, $T \ll T_{K}^{(j)}$, the transmission amplitude exhibits the typical Kondo behavior: in the local-moment regime a typical Kondo plateau forms, with $\left|t_{d}\right|$ approaching the unitary limit, $\left|t_{d}\right| \rightarrow 1$. In the mixed valence regime the magnitude changes rapidly as a function of $V_{g}$. In the Coulomb blockade valleys, transmission is suppressed by Coulomb interaction. The transmission phase increases by $\sim \pi / 2$ for each entering electron (see black curves for $\alpha$ in Fig. 11), increasing only slightly in between. In the Kondo valleys this results in a plateau at $\alpha \bmod \pi=\frac{\pi}{2}$, as direct consequence of the $\frac{\pi}{2}$ phase shift due to the formation of the Kondo singlet.

With increasing temperature, the Kondo effect is suppressed, thus the behavior in the middle of the Kondo valleys changes dramatically. The Kondo plateaus in $t_{d}$ and $\alpha$ disappear: The magnitude tends towards Coulomb blockade behavior, with a resonance of width $\sim \Gamma_{j}$ for each entering electron. The phase develops a $S$-like shape in the Kondo valleys with increasing temperature. As in the single-level case, all finite-temperature curves of the phase intersect the zero-temperature at the same gate voltage, say $V_{g}^{c_{j}}$ (see red arrows). We shall refer to this gate voltage as a "stationary" point (w.r.t. temperature).

As observed in the experiments of $\mathrm{Ji}$ et $a l^{\underline{\underline{3}}}$ and

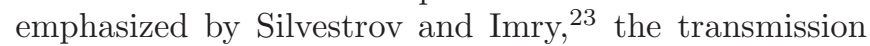
phase reacts more sensitively to the buildup of Kondo correlations with decreasing temperature than the transmission magnitude: $\alpha$ approaches its $T=0$ behavior already at temperatures $T \simeq T_{K}$ (the $\frac{\pi}{2}$-plateau develops), whereas $\left|t_{d}\right|$ develops its plateau for $T$ significantly less than $T_{K}$ (see, the green curve $\left(T / U=10^{-4}\right)$ for level 1 in Fig. 1(b) or the purple curve $\left(T / U=10^{-6}\right)$ for level 2 in Fig. 1(a)). Similar to the predictions of Silvestrov and Imry, $\stackrel{23}{=}$ we find shoulders in the evolution of the phase, see for example the fat purple arrow and the purple curve $\left(T / U=10^{-6}\right)$ in Fig. 1(c). This indicates that the temperature is large enough to suppress Kondo correlations in the deep local-moment regime (in the middle of the Kondo valley), where $T_{K}$ is very small. Towards the borders of the local-moment regime the crossover temperature for the onset of phase sensitivity increases (as does the Kondo temperature, see Eq. (6)), eventually exceeding the temperature. Then the phase tends towards its zero-temperature behavior, thus producing shoulders.

Properties special to the multi-level model: The most obvious difference between the transmission amplitude of the many-level model in the mesoscopic regime compared to the single-level model is the phase behavior in the Coulomb blockade valleys between the levels. Depending on $s$, i.e. on the relative sign of the tunnelling matrix elements of the two adjacent levels, the phase either exhibits a sharp drop (phase lapse) by $\pi$ in the $s=+$ case (accompanied by a transmission zero, $\left|t_{d}\right|=0$ ), or evolves continuously for $s=-11,12,14,24,25,26$. Contrary to the non-monotonic phase evolution discussed above, this ef- fect occurs already at zero temperature and also exists for spinless models $\stackrel{11,12}{12}$ Therefore, the relevant energy scale for the temperature dependence of this phase lapse is not related to the Kondo temperature but to the level distance and width of the effective transport levels $\frac{23}{\stackrel{23}{ } \text { It is }}$ therefore not a relevant energy scale in the temperature range studied in this work.

A further peculiarity for models with more than one level is the asymmetry (w.r.t. the center of the Kondo valleys) of the transmission amplitude in the local-moment regime at finite temperature, introduced by the mixing of neighboring levels. The asymmetry in phase can be characterized by the position of the stationary points, $V_{g}^{c_{j}}$ (indicted by red arrows in Fig. 11). In case $s=+$, these points are repelled by the neighboring level, whereas they are shifted to the latter for $s=-$, compare for example Fig. 1(a) and (c) or (b) and (d). For $\Gamma_{1} / \Gamma_{2} \neq 1$, the repulsion and attraction is enhanced or reduced compared to $\Gamma_{1}=\Gamma_{2}$ for the level that is coupled less or more strongly to the leads, respectively. Clearly, in the limit of one decoupled level (effective one-level system), the stationary point of the other level is symmetric w.r.t. the corresponding Kondo plateau. The dips that form in the plateaus of the amplitude with increasing temperature develop a distinct asymmetry only for $T \gg T_{K}^{(j)}$, for which they tend to shift towards the corresponding $V_{g}^{c_{j}}$. This is consistent with the fact that as the phase drop in the Kondo valley gets sharper with increasing temperature and approaches a quasi-phase lapse, the magnitude experiences a minimum, as for every complex function. Interestingly, the asymmetry in phase is the same for all temperatures, thus already at temperature $T \lesssim T_{K}$ the phase "knows" in which direction (of $V_{g}$ ) the dip in magnitude will shift at higher temperatures.

\section{B. Dependence on the coupling strength}

In experiments, it is more convenient (and easier to control) to change the coupling strength between the quantum dot and the reservoirs than the temperature. Accordingly, Fig. 2 presents the transmission amplitude for various values of $\Gamma$, keeping the temperature constant. With decreasing $\Gamma$, the decrease of $T_{K}$ together with the suppression of Kondo correlations is nicely illustrated. At fixed temperature $T>T_{K}$, the S-like shape of the phase evolution gets more pronounced and sharper with decreasing $\Gamma$.

In the single-level problem, in addition to stationary points w.r.t. temperature, we also find stationary points w.r.t. $\Gamma$ for $t_{d}$, i.e. for magnitude and phase of the transmission amplitude. These occur at the outer flanks of the Kondo plateaus. Varying the mean coupling strength $\Gamma$ at fixed $\gamma, \delta$ and $T$ in the two-level model, as shown in Fig. 2. these points can still be recognized (indicated by green arrows in (d)), even though the $\Gamma$-independence 


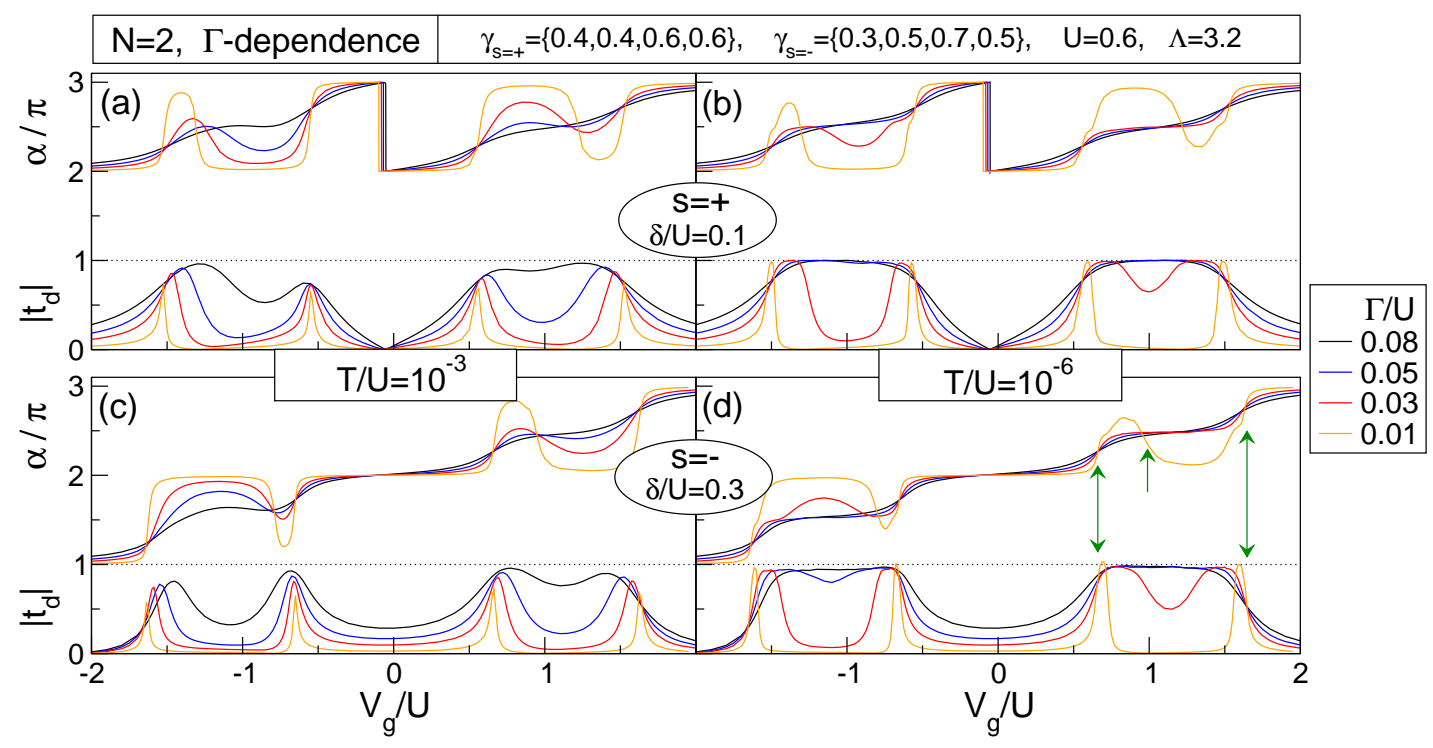

FIG. 2: Transmission through a spinful two-level quantum dot for both choices of $s= \pm$ and various values of mean couplings $\Gamma$ at fixed temperature $T$, level spacing $\delta$ and relative couplings $\gamma$. Due to the mixing of the levels, no stationary points w.r.t. $\Gamma$ exist, $\Gamma$-independence exist, see text and the green arrows in $(d)$.

is not perfect (within our numerical accuracy). We expect that due to the mixing of the levels, also the level distance $\delta$ has to be taken into account to recover these stationary points. Between the levels, near $V_{g} / U \approx 0$, another stationary point seems to occur.

\section{Three-level model}

Naturally, the question arises about the effects of several levels, with different choices of $s_{i}= \pm$, which is present only for models with more than two levels. Assuming that in the mesoscopic regime only neighboring levels mix significantly, i.e. simultaneously influence transport, any local level of a quantum dot (except the lowest or highest one) can be represented adequately by the middle level of a three-level model.

In Fig. 3 we present numerical data of a three-level model for all four possible combinations of $s=s_{1}, s_{2}$ and various temperatures. The second level is influenced by the effect of both $s_{1}$ and $s_{2}$, resulting in an effective enhancement or compensation of the asymmetry of the stationary point $V_{g}^{c_{2}}$ of level 2, as discussed in Section III A 1. Also the relative strength of the level-couplings (given by $\gamma$ ) has to be considered. In Fig. 3(a), both $s$ and $\gamma$ symmetrize the transmission curves of the middle level, whereas in panel 3(b) $\gamma$ shifts $V_{g}^{c_{2}}$ to positive $V_{g}$. In panels 3(c) and 3(d) both $s$ and $\gamma$ tend to increase the asymmetry.

Therefore, the transmission phase through a spinful quantum dot with Kondo correlations present has $S$-like shape in the local-moment regimes at $T \gg T_{K}$. Analogously to experiments, we find an asymmetry of this $S$ - like shape. It is determined by both the relative strength $\gamma$ and the sign $s$ of the level couplings.

\section{CONCLUSION}

In this paper we present temperature-dependent NRG calculations of the magnitude and phase of the transmission amplitude through a multi-level quantum dot in the regime $\delta / \Gamma \ll 1$. Clearly, the Kondo correlations are suppressed with increasing temperature. The presence of neighboring levels results in a $V_{g}$-asymmetry in the finite temperature modulation of the Kondo valleys. The asymmetry depends on the relative signs of the tunneling matrix elements as well as on the relative couplings of the adjacent levels. Further, sharp phase lapses may occur between the levels. Studying a three-level model, the middle level can be understood as a representative of a generic level in a multi-level quantum dot.

Throughout the paper, we deliberately focussed only on the deep mesoscopic regime, for which the results can be understood rather straightforwardly. The crossover into the regime $\delta / \Gamma \simeq 1$, which is certainly of interest too in order to understand the fate of Kondo physics in the universal regime, and which we believe to be the regime relevant for the experiments of Ji et al. $\stackrel{3,4}{\underline{3}}$ will be left as a subject for future studies.

\section{ACKNOWLEDGEMENT}

We acknowledge helpful discussions with Moty Heiblum, Michele Zaffalon, Vitaly Golovach and Michael Pustilnik. This research was supported by the DFG 


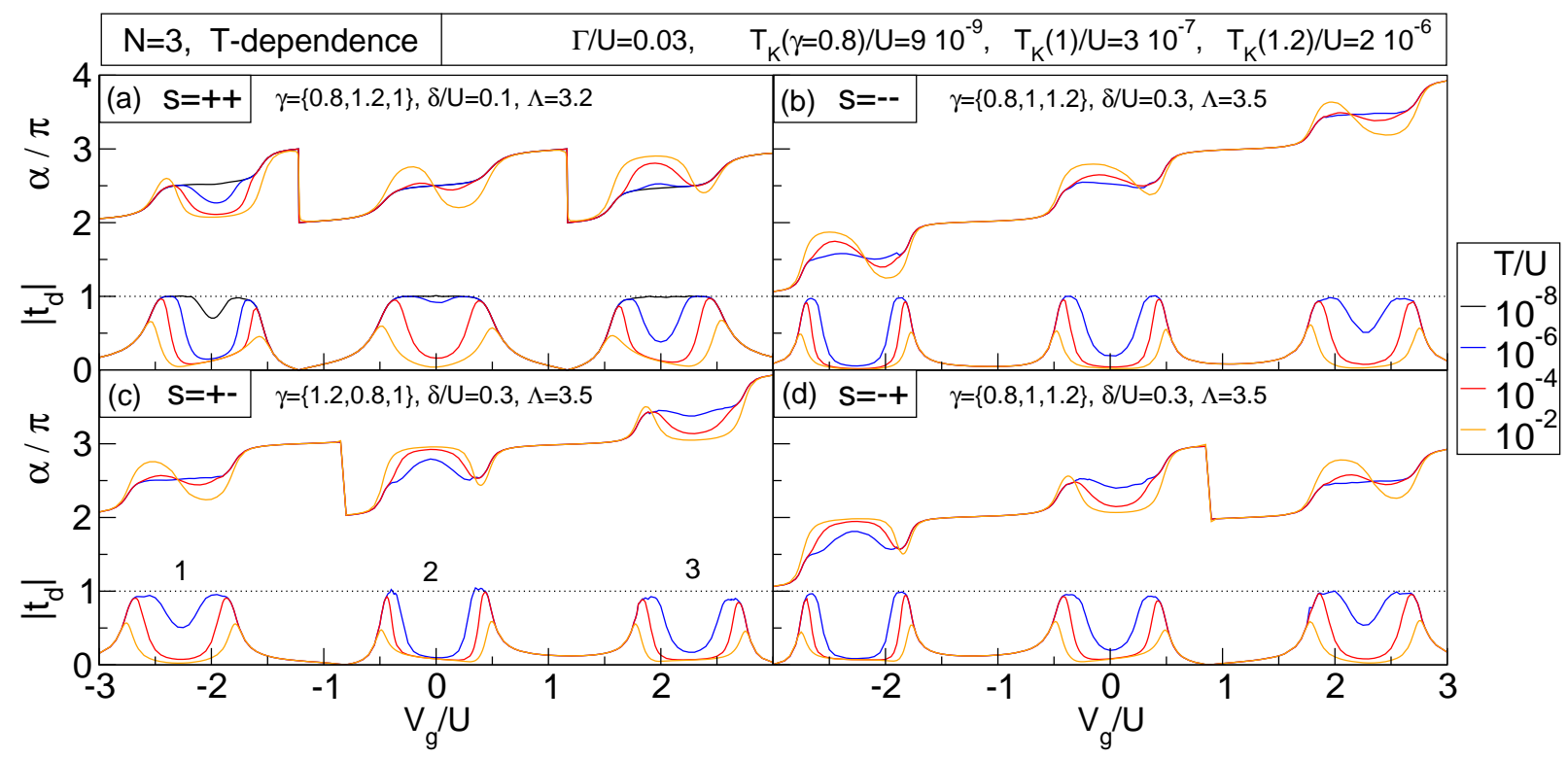

FIG. 3: Transmission through a three-level model for all four possible combinations of $s=s_{1}, s_{2}$ and various temperatures, for fixed $\Gamma / U=0.03$. The two-channel calculations for (b,c,d) qualitatively capture the physical trends. The asymmetry in the Kondo valleys is determined by both $s$ and $\gamma$. For convenience the figure legends for $\gamma$ display only the total relative coupling of each level. The minimal bare Kondo temperatures are indicated. (a) $s=++: \gamma=\{0.6,0.6,0.4,0.4,0.5,0.5\}$. The case $T / U=$ $10^{-8}$ is included only for this panel. (b) $s=--: \gamma=\{0.5,0.3,0.4,0.6,0.5,0.7\}$. (c) $s=+-: \gamma=\{0.4,0.8,0.4,0.4,0.3,0.7\}$. (d) $s=-+: \gamma=\{0.5,0.3,0.3,0.7,0.7,0.5\}$.

through De-730/3-2, SFB631 and SFB-TR12, and by DIP-H.2.1. Financial support of the German Excellence Initiative via the "Nanosystems Initiative Munich (NIM)" is gratefully acknowledged.

\section{APPENDIX A: CONDUCTANCE FORMULA FOR MULTI-TERMINAL GEOMETRY}

\section{General case}

We generalize the current formula derived in by Bruder, Fazio and Schoeller ${ }^{14}$ for a single-level quantum dot embedded into one arm of an Aharonov-Bohm interferometer with two-terminal geometry to a multiterminal geometry with a multi-level dot (as used in the Heiblum group $2,3,4,5,6$ ).

Consider a $N$-level quantum dot described by $H_{\mathrm{d}}[\mathrm{Eq}$. (1b)] embedded in one arm of an Aharonov-Bohm interferometer connected to $M$ leads, as depicted in Fig. [4 Each lead, and each arm connecting them, is assumed to support only a mode. The tunnelling between the local levels $j=1 \cdots N$ on the quantum dot and the leads $\alpha=1 \cdots M$ is described by

$$
H_{\mathrm{t}}=\sum_{j \sigma} \sum_{\alpha k} t_{\varepsilon \alpha \sigma}^{j} c_{\alpha \varepsilon \sigma}^{\dagger} d_{j \sigma}+\text { H.c. }
$$

Here $t_{\varepsilon \alpha \sigma}^{j}=\sum_{i=L, R} t_{i \sigma}^{j} A_{\varepsilon \alpha \sigma}^{i}$ (indicated in green in Fig.
(4) is chosen real, where $t_{i \sigma}^{j}=\left\langle x_{i} \mid j \sigma\right\rangle$ (blue) is the amplitude to get from dot state $|j \sigma\rangle$ of level $j$ and spin $\sigma$ to point $x_{i}$ on side $i=L, R$ of the dot, and $A_{\varepsilon \alpha \sigma}^{i}=\left\langle\varepsilon \alpha \sigma \mid x_{i}\right\rangle$ (red) is the amplitude to get from point $x_{i}$ to lead state $|\varepsilon \alpha \sigma\rangle$ in lead $\alpha$ with energy $\varepsilon$ and spin $\sigma$, see Fig. 4

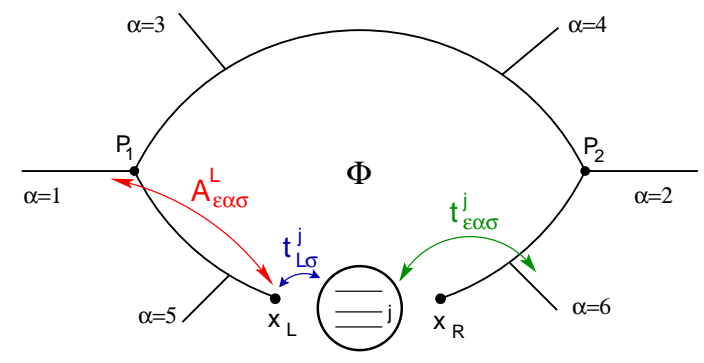

FIG. 4: Geometry of the multi-terminal Aharonov-Bohm interferometer with a multi-level quantum dot embedded in the lower arm. The different tunnelling amplitudes used in the text are indicated. $\Phi$ is the magnetic flux penetrating the interferometer.

Following Büttiker, $\stackrel{27}{\rightleftharpoons}$ the current operator in reservoir $\alpha$ is given by

$$
\hat{I}_{\alpha}(t)=\frac{e}{h} \sum_{\varepsilon \varepsilon^{\prime}} \sum_{\sigma \sigma^{\prime}} \frac{1}{\rho}\left[c_{\varepsilon^{\prime} \alpha \sigma^{\prime}}^{\dagger}(t) c_{\varepsilon \alpha \sigma}(t)-b_{\varepsilon^{\prime} \alpha \sigma^{\prime}}^{\dagger}(t) b_{\varepsilon \alpha \sigma}(t)\right],
$$

where $\rho$, the density of states, is assumed to be constant and equal for each reservoir. The first term inside the bracket stands for the incident, the second term for the 
reflected current in reservoir $\alpha$, thus $b_{\varepsilon \alpha \sigma}=\sum_{\beta} S_{\alpha \beta}^{\varepsilon} c_{\varepsilon \beta \sigma}$, with $S_{\alpha \beta}^{\varepsilon}$ the scattering amplitude to get from lead $\beta$ to lead $\alpha$ with energy $\varepsilon$. Defining the lesser, retarded and advanced correlation functions

$$
\begin{aligned}
\mathcal{G}_{\mu, \mu^{\prime}}^{<}\left(t-t^{\prime}\right) & \equiv \frac{i}{\hbar}\left\langle a_{\mu^{\prime}}^{\dagger}\left(t^{\prime}\right) a_{\mu}(t)\right\rangle \\
& =\int \frac{d E}{2 \pi \hbar} e^{-i E\left(t-t^{\prime}\right) / \hbar} \mathcal{G}_{\mu, \mu^{\prime}}^{<}(E), \\
\mathcal{G}_{\mu, \mu^{\prime}}^{R, A}\left(t-t^{\prime}\right) & \equiv-\frac{i}{\hbar} \theta\left( \pm\left(t-t^{\prime}\right)\right)\left\langle\left[a_{\mu^{\prime}}^{\dagger}\left(t^{\prime}\right), a_{\mu}(t)\right]_{+}\right\rangle \\
& =\int \frac{d E}{2 \pi \hbar} e^{-i E\left(t-t^{\prime}\right) / \hbar} \mathcal{G}_{\mu, \mu^{\prime}}^{R, A}(E),
\end{aligned}
$$

where $a_{\mu}$ denotes a fermionic operator with composite index $\mu$, the expectation value of the current operator (A2) can be expressed as

$$
\begin{aligned}
\left\langle\hat{I}_{\alpha}(t)\right\rangle= & \frac{e}{h} \sum_{\varepsilon \varepsilon^{\prime}} \sum_{\beta \beta^{\prime}} \sum_{\sigma \sigma^{\prime}} \frac{1}{\rho}\left[\delta_{\alpha \beta^{\prime}} \delta_{\alpha \beta}-S_{\alpha \beta^{\prime}}^{\star \varepsilon^{\prime}} S_{\alpha \beta}^{\varepsilon}\right] \\
& \times(-i) \int \frac{d E}{2 \pi} \mathcal{G}_{\varepsilon \beta \sigma, \varepsilon^{\prime} \beta^{\prime} \sigma^{\prime}}^{<}(E) .
\end{aligned}
$$

To calculate $\mathcal{G}^{<}(E)$ in Eq. A5 , we use the standard Dyson equation for the Keldysh $2 \times 2$ matrix Green's function $^{28} \hat{\mathcal{G}}(E)$,

$$
\begin{aligned}
& \hat{\mathcal{G}}_{\varepsilon \alpha \sigma, \varepsilon^{\prime} \alpha^{\prime} \sigma^{\prime}}(E)=\delta_{\varepsilon \varepsilon^{\prime}} \delta_{\alpha \alpha^{\prime}} \delta_{\sigma \sigma^{\prime}} \hat{\mathcal{G}}_{\varepsilon \alpha \sigma}^{0}(E) \\
& \quad+\sum_{j j^{\prime}} \hat{\mathcal{G}}_{\varepsilon \alpha \sigma}^{0}(E) t_{\varepsilon \alpha \sigma}^{j} \hat{\mathcal{G}}_{j \sigma, j^{\prime} \sigma^{\prime}}^{d}(E) t_{\varepsilon^{\prime} \alpha^{\prime} \sigma^{\prime}}^{\star j^{\prime}} \hat{\mathcal{G}}_{\varepsilon^{\prime} \alpha^{\prime} \sigma^{\prime}}^{0}(E),
\end{aligned}
$$

which yields

$$
\begin{aligned}
\mathcal{G}_{\varepsilon \alpha \sigma, \varepsilon^{\prime} \alpha^{\prime} \sigma^{\prime}}^{<}(E) & =\delta_{\varepsilon \varepsilon^{\prime}} \delta_{\alpha \alpha^{\prime}} \delta_{\sigma \sigma^{\prime}} \mathcal{G}_{\varepsilon \alpha \sigma}^{0<}(E) \\
& +\sum_{j j^{\prime}} t_{\varepsilon \alpha \sigma}^{j}[A+B+C] t_{\varepsilon^{\prime} \alpha^{\prime} \sigma^{\prime}}^{\star j^{\prime}},
\end{aligned}
$$

where terms in square brackets are given by

$$
\begin{aligned}
& A=\mathcal{G}_{\varepsilon \alpha \sigma}^{0 R}(E) \mathcal{G}_{j \sigma, j^{\prime} \sigma^{\prime}}^{R}(E) \mathcal{G}_{\varepsilon^{\prime} \alpha^{\prime} \sigma^{\prime}}^{0<}(E), \\
& B=\mathcal{G}_{\varepsilon \alpha \sigma}^{0 R}(E) \mathcal{G}_{j \sigma, j^{\prime} \sigma^{\prime}}^{<}(E) \mathcal{G}_{\varepsilon^{\prime} \alpha^{\prime} \sigma^{\prime}}^{0 A}(E), \\
& C=\mathcal{G}_{\varepsilon \alpha \sigma}^{0<}(E) \mathcal{G}_{j \sigma, j^{\prime} \sigma^{\prime}}^{A}(E) \mathcal{G}_{\varepsilon^{\prime} \alpha^{\prime} \sigma^{\prime}}^{0 A}(E),
\end{aligned}
$$

where the free Green's functions for the leads have the form

$$
\begin{aligned}
\mathcal{G}_{\varepsilon \alpha \sigma}^{0 R, A}(E) & =\frac{1}{E-\varepsilon \pm i O^{+}}, \\
\mathcal{G}_{\varepsilon \alpha \sigma}^{0<}(E) & =2 \pi i f_{\alpha}(E) \delta(\varepsilon-E),
\end{aligned}
$$

with $f_{\alpha}(E)$ the Fermi function of lead $\alpha$. Inserting $\mathcal{G}^{<}$ [Eq. (A7)] into Eq. (A5), the current can be written as

$$
\left\langle\hat{I}_{\alpha}\right\rangle=I_{\alpha}^{0}+\delta I_{\alpha}
$$

$I_{\alpha}^{0}$ arises from the first term of Eq. A7). It describes the situation when the dot is completely decoupled $\left(t_{\varepsilon \alpha \sigma}^{j}=0\right)$, thus does not contribute to Aharonov-Bohm oscillations. The influence of the quantum dot on the Aharonov-Bohm oscillations is caused by $\delta I_{\alpha}$, arising from the second expression of Eq. A7). Performing the energy sums $\sum_{\varepsilon \varepsilon^{\prime}}$ in Eq. A10 or A5 , respectively, the two contributions to the current read

$$
\begin{aligned}
I_{\alpha}^{0} & =\frac{e}{h} \int d E \sum_{\sigma} \sum_{\beta}\left[\delta_{\alpha \beta}-\left|S_{\alpha \beta}^{E}\right|^{2}\right] f_{\beta}(E), \quad(\mathrm{A} 11 \\
\delta I_{\alpha} & =\frac{e}{h} \operatorname{Re}\left\{\int d E \sum_{\beta \beta^{\prime}} \sum_{j j^{\prime}} \sum_{\sigma \sigma^{\prime}}\left[\delta_{\alpha \beta^{\prime}} \delta_{\alpha \beta}-S_{\alpha \beta^{\prime}}^{\star E} S_{\alpha \beta}^{E}\right]\right. \\
& \times \pi \rho t_{E \beta \sigma}^{j} t_{E \beta^{\prime} \sigma^{\prime}}^{\star j^{\prime}}(-i) \\
& \left.\times\left[2 \mathcal{G}_{j \sigma, j^{\prime} \sigma^{\prime}}^{R}(E) f_{\beta^{\prime}}(E)+\mathcal{G}_{j \sigma, j^{\prime} \sigma^{\prime}}^{<}(E)\right]\right\} .
\end{aligned}
$$

\section{Simplification to effective 2-lead geometry}

For the experimental setup used by Schuster et $a l_{\text {. }}^{2}$ (and equivalently for the ensuing papers $3,4,5,6$ ) to measure transmission phase shifts, two simplifying assumptions can be made. The first allows us to neglect non equilibrium effects, the second to perform NRG calculations for a simplified geometry, in which the dot is coupled only to two leads.

(i) Neglect of non equilibrium effects: In the experimental setup used by Schuster et al., 2 the leads $\alpha=3,4,5$ and 6 serve as draining reservoirs (to prevent multiple traversals of the ring, see below), and are all kept at chemical potential $\mu_{\alpha}=0$. This also fixes the chemical potential of the ring, referred to as "base region" in Ref. 2 , to equal zero. Lead 1 and 2 serve as emitter and collector, respectively, with chemical potentials $\mu_{1}$ and $\mu_{2}$, and Fermi functions $f_{1,2}(E)=f_{0}\left(E-\mu_{1,2}\right)$. Now, the point contacts between emitter or collector and the base region (marked $P_{1}$ and $P_{2}$ in Fig. 4) are so small that the voltage drops occur directly at these point contacts, and not at the tunnel barriers coupling the dot to the ring. Thus, while the emitter or collector inject or extract electrons into or from the base region, respectively, this is assumed to happen at a sufficiently small rate that the base region is not disturbed. In other words, we may assume that the dot, ring, and electrodes 3, 4, 5, 6 are all in equilibrium with each other, and that the dot Green's functions $\mathcal{G}_{j \sigma, j^{\prime} \sigma^{\prime}}^{R, A,<}(E)$ do not depend on $\mu_{1}$ and $\mu_{2}$ at all. Thus, the lesser function can be expressed in terms of the retarded and advanced ones using the following standard equilibrium relation:

$$
\mathcal{G}_{j \sigma, j^{\prime} \sigma^{\prime}}^{<}(E)=-f_{0}(E)\left[\mathcal{G}_{j \sigma, j^{\prime} \sigma^{\prime}}^{R}(E)-\mathcal{G}_{j \sigma, j^{\prime} \sigma^{\prime}}^{A}(E)\right] .
$$

The conductance in the linear response regime can be obtained by taking $\mu_{1}-\mu_{2}=e V$, where $e=|e|$, e.g. by setting

$$
\mu_{1}=0, \quad \mu_{2}=-e V
$$

and calculating $G=\partial I_{1} / \partial V$, with $I_{1}$ given by Eq. A11. 
(ii) Reduction to two-lead geometry: The reason why a multi-lead geometry was used in experiment is to avoid phase-rigidity: in an Aharonov-Bohm ring connected to only two leads, the transmission phase of the dot does not vary smoothly with gate voltage, but can assume only two distinct values, differing by $\pi$. A multi-lead geometry avoids this by strongly reducing the probability amplitudes for paths from emitter to collector to traverse the ring multiple times, since with each traversal of the ring the probability increases that electrons travelling in the ring are "siphoned off" into the side arms. We shall exploit this fact by making the assumption that the probability amplitudes for multiple traversals of the ring are negligibly small. This assumption allows us to replace the multi-lead geometry with one where the Aharonov-Bohm ring is coupled to only two leads, i.e. $\alpha$ is restricted to the values 1 and 2 (corresponding to emitter and collector), while multiple traversals of the ring are eliminated (by hand) by the following specification: The amplitude $t_{\varepsilon \alpha \sigma}^{j}$ to get from state $|j \sigma\rangle$ on the dot to state $|\varepsilon \alpha \sigma\rangle$ in lead $\alpha$ is taken to be nonzero only for the short, direct path from the dot to lead $\alpha$, without traversing the upper arm (more correctly: we take $A_{\varepsilon \alpha \sigma}^{L}=0$ for $\alpha=2,4,6$ and $A_{\varepsilon \alpha \sigma}^{R}=0$ for $\left.\alpha=1,3,5\right)$. When calculating the current we do allow for direct paths from lead 1 to 2 via the upper arm, and lump all flux-dependence into the corresponding scattering amplitude, taking $S_{12}^{E} \sim e^{i 2 \pi \Phi / \Phi_{0}}$. However, the upper arm is ignored for the calculation of the equilibrium local retarded or advanced Green functions $\mathcal{G}_{j \sigma, j^{\prime} \sigma^{\prime}}^{R, A}(E)$ using NRG. For the latter purpose, we thus use a model of a multi-level dot coupled to two independent leads, say $L$ and $R$, with equal chemical potentials $\mu_{L}=\mu_{R}$, representing the two segments of the ring to the left and right of the ring, coupled to it by tunnelling contacts. (These two segments should be treated as independent leads, due to the assumption of no multiple traversals made above.) With the assumptions (i) and (ii) just described, let us now obtain an expression for that part of the conductance showing Aharonov-Bohm oscillations with applied flux, $G^{A B}=\frac{\partial I_{1}^{A B}}{\partial V}$, where $I_{1}^{A B}$ is that part of the current in lead 1 depending on $e^{i 2 \pi \Phi / \Phi_{0}}$. For the chemical potentials given by Eq. (A13), this corresponds to evaluate Eq. A11b with $\alpha=1, \beta^{\prime}=2$ and $\beta=1$, and we readily obtain

$$
G^{A B}(T)=\frac{e^{2}}{h} \int d E \operatorname{Re}\left[T_{u}^{\star}(E) T_{d}(E)\right]\left(-\frac{\partial f_{0}(E)}{\partial E}\right),
$$

where

$$
\begin{aligned}
T_{d}(E) & =\sum_{j j^{\prime}} \sum_{\sigma \sigma^{\prime}} 2 \pi \rho t_{E 1 \sigma}^{j} \mathcal{G}_{j \sigma, j^{\prime} \sigma^{\prime}}^{R}(E) t_{E 2 \sigma^{\prime}}^{\star j^{\prime}} \\
T_{u}^{\star}(E) & =i S_{12}^{\star E} S_{11}^{E}=\left|T_{u}(E)\right| e^{i\left(2 \pi \Phi / \Phi_{0}+\phi_{0}(E)\right)}
\end{aligned}
$$

may be interpreted as the transmission amplitudes through the lower and upper arms, respectively.

Assuming the transmission amplitude $T_{u}$ through the upper arm to be energy- and temperature-independent, the Aharonov-Bohm contribution to the conductance is given by

$$
G^{A B}(T)=\frac{e^{2}}{h} T_{u}|| t_{d}(T) \mid \cos \left(2 \pi \Phi / \Phi_{0}+\phi_{0}+\alpha(T)\right) .(A 1
$$

Then, the temperature-dependent magnitude and phase of the transmission amplitude through the quantum dot,

$$
t_{d}(T)=\int d E\left(-\frac{\partial f_{0}(E, T)}{\partial E}\right) T_{d}(E, T) \equiv\left|t_{d}(T)\right| e^{i \alpha(T)},
$$

can be (i) extracted via Eq. A17) from the experimental results as well as (ii) calculated with NRG using Eq. (A15).
1 A. Yacoby, M. Heiblum, D. Mahalu and H. Shtrikman, Phys. Rev. Lett. 74, 4047 (1995).

2 R. Schuster, E. Buks, M. Heiblum, D. Mahalu, V. Umansky and H. Shtrikman, Nature 385, 417 (1997).

3 Y. Ji, M. Heiblum, D. Sprinzak, D. Mahalu and H. Shtrikman, Science 290, 779 (2000).

4 Y. Ji, M. Heiblum and H. Shtrikman, Phys. Rev. Lett. 88, 076601 (2002).

${ }^{5}$ M. Avinun-Kalish, M. Heiblum, O. Zarchin, D. Mahalu and V. Umansky, Nature 436, 529 (2005).

${ }^{6}$ M. Zaffalon, B. Aveek, M. Heiblum, D. Mahalu and V. Umansky, cond-mat/0711.1172 (2007).

7 U. Gerland, J. von Delft, T. A. Costi and Y. Oreg, Phys. Rev. Lett. 84, 3710 (2000).

8 P. G. Silvestrov and Y. Imry, Phys. Rev. Lett. 85, 2565 (2000).

9 P. G. Silvestrov and Y. Imry, Phys. Rev. B 65, 035309 (2001).

10 D. I. Golosov and Y. Gefen, Phys. Rev. B 74, 205316
(2006).

11 C. Karrasch, T. Hecht, A. Weichselbaum, Y. Oreg, J. von Delft and V. Meden, Phys. Rev. Lett. 98, 186802 (2007).

12 C. Karrasch, T. Hecht, A. Weichselbaum, J. von Delft, Y. Oreg and V. Meden, New J. Phys. 9, 123 (2007).

13 Y. Oreg, New J. Phys. 9, 122 (2007).

14 C. Bruder, R. Fazio and H. Schoeller, Phys. Rev. Lett. 76, 114 (1996).

15 M. Pustilnik and L. I. Glazman, Phys. Rev. Lett. 87, 216601 (2001).

16 D. C. Langreth, Phys. Rev. 150, 516 (1966).

17 H. R. Krishna-murthy, J. W. Wilkins and K. G. Wilson, Phys. Rev. B 21, 1003 (1980).

18 R. Bulla, T. A. Costi and T. Pruschke, Rev. Mod. Phys. 80, 395 (2008).

19 R. Peters, T. Pruschke and F. B. Anders, Phys. Rev. B 74, 245114 (2006).

20 A. Weichselbaum and J. von Delft, Phys. Rev. Lett. 99, 076402 (2007). 
21 F. B. Anders and A. Schiller, Phys. Rev. Lett. 95, 196801 (2005).

22 R. Bulla, A. C. Hewson and T. Pruschke, J. Phys.: Condens. Matter 10, 8365 (1998).

23 P. G. Silvestrov and Y. Imry, Phys. Rev. Lett. 90, 106602 (2003).

24 Y. Oreg and Y. Gefen, Phys. Rev. B 55, 13726 (1997).
25 A. L. Yeyati and M. Büttiker, Phys. Rev. B 52, R14360 (1995).

26 G. Hackenbroich, Phys. Rep. 343, 463 (2001).

27 M. Büttiker, Phys. Rev. B 46, 12485 (1992).

28 J. Rammer and H. Smith, Rev. Mod. Phys. 58, 323 (1986). 